\title{
A défaut de dire tout: dire partout. Étude des modes énonciatifs dans Le mespris de la vie et consolation

\author{
contre la mort de \\ Jean-Baptiste Chassignet
}

ISABELLE LACHANCE

Résumé: Poésie maniériste? Baroque? Le mespris de la vie et consolation contre la mort (1594) peut-être le résultat de multiples influences, et chaque situation d'énonciation de ce recueil comporte sa manière propre de structurer les courants idéologiques de l'époque à laquelle il a été écrit, et ce, dans un but didactique d'illustration, comme le précise d'ailleurs la "Preface au lecteur." Les Essais sont sûrement l'une de ces influences. Toutefois, alors que chez Montaigne le moi constitue "une médiation pour connaître le monde," chez Chassignet, le "je" est au service de l'illustration. On aura beau chercher à tort et à travers la "mélancolie de Chassignet"2 ou l'expression de son "moi clivé," le seul affect qu'il est possible de reconstituer à la lecture du recueil est celui du sujet partout dépeint comme la figure exemplaire du pécheur.

n a déjà qualifié les poèmes de Chassignet de "lourdes rhapsodies, prolixes et souvent banales." 4 Après une première lecture du Mespris de la vie et consolation contre la mort, on a certainement l'impression d'avoir entendu et réentendu deux ou trois idées principales. Par conséquent, je ne m'attarderai pas seulement à commenter le contenu de cette oeuvre, mais surtout à rendre compte de la manière dont on y prend la parole, sans prendre le parti exclusivement formel de la considérer comme maniériste (parce qu'elle ressasse infiniment les mêmes idées), affirmant par-là qu'elle témoigne de "la désagrégation [. . . ] des théories de la littérature et de l'art" ou qu'elle remet en cause "la pratique artistique" et ses "normes idéales"5 
en privilégiant la maniera sur le contenu. En effet, la lecture de la "Preface au lecteur" suffit à convaincre que l'intention de l'oeuvre est clairement illustrative et didactique:

[. . . ] je conclus en moy-mesme de [...] te monstrer, amy lecteur, l'infirmité et misere de nostre condition par le premier trait de ce discours, comme le peintre monstre l'excellence de son art sous les crayons d'un portrait [... . ${ }^{6}$

[...] retastant et pestrissant ceste nouvelle matiere, la remuant et reschauffant, ouvrir a celuy qui la voudra traitter à fond de cuve, quelque facilité pour en jouir plus à son aise, la luy rendant plus souple et maniable (Mespris, p. 20).

On ne peut donc garder sous silence l'apport d'un si imposant recueil à l'art post-tridentin, instrument d'une Église romaine qui "réaffirme, par le moyen du baroque, la valeur idéale et la nécessité pratique de la démonstration [... .], en vue de l'édification"7: il s'agit d'un art qui "exerce un effet direct" et qui doit "se rend[re] utile."8

"L'Orateur et le Poète [sont] tant proches et conjoints,"9 nous dit Sébillet dans son Art poëtique, et c'est ce que montre François Cornilliat qui conclut que, pour cette période, "l' autonomie du poème par rapport aux autres genres du discours est relativisée." 10 C'est en regard de la même observation que j'ai voulu aborder le Mespris par l'approche énonciative, afin de comprendre comment s'entrecroisent ou se confrontent les influences que l'on peut y rencontrer. De plus, cette approche parvient à joindre l'observation structurale à la contextualisation historique: en effet, la perspective énonciative a ceci de positif qu'elle "dénonce le leurre représenté par le postulat de la clôture structurale du texte et de l'énoncé," "ll énoncé qui devient une réalité déterminée par sa "condition contextuelle de production / réception." 12

On peut comprendre les réserves de la critique - plus particulièrement celles de Terence Cave lorsqu'il affirme que "Chassignet fails in his philosophical sonnets because he is unable to adapt serious thoughts to the needs of a poem: his arguments always have the air of imported fragments"13 — à l'égard du traitement un peu paradoxal des idées dans les sonnets plus philosophiques du Mespris. En effet, cette poésie est tributaire d'une part de valeurs religieuses quasi médiévales qui posent que le vrai chrétien doit vivre hors du monde et, d'autre part, du vent de néo-stoïcisme qui souffle sur le dernier quart du seizième siècle et qui se veut une philosophie de l'homme dans le monde, comme en témoignent le traité De la constance de Juste Lipse ou le Discours de la vie et de la mort de Philippe Du Plessis-Mornay. ${ }^{14}$ Or, si l'on considère la volonté pédagogique signifiée dans la "Preface," l'aspect 
éclaté du Mespris ne résulterait pas tant d'une maiadresse que de la démonstration objective, dans ces sonnets où prime le discours à la troisième personne, de plusieurs options idéologiques.

\section{Le lieu de la "non-personne"}

Tentant de repérer les marques de la subjectivité dans toute production langagière, il est bien sûr possible de retenir comme indice le caractère déictique sous-jacent à toute prise de parole. Cependant, opter pour cette orientation dans le cadre d'une lecture des poèmes rédigés à la troisième personne dans le Mespris serait déroger à un certain pacte proposé par le locuteur. En effet, utilisant une forme qui le place d'emblée hors de la situation d'énonciation, il place son discours sous le mode de l'objectivité, ${ }^{15}$ du moins de l'idée plus que de l'expression lyrique ou subjective. Dans ce type d'énoncé, la présence du sujet de l'énonciation peut se voir avantageusement repérée par l'observation des présuppositions et connotations qui orientent l'énoncé, ainsi que par l'examen de l'image du destinataire qu'il propose. Par exemple, un titre tel que Vie et mort correspond à une suite événementielle sinon universelle dans l'imaginaire, du moins commune. Cependant, le titre du recueil de Chassignet impose d'emblée un contenu connotatif précis: poser le "mépris de la vie" implique l'existence d'un univers discursif qui conçoit la vie comme un objet estimable contre lequel s' inscrira le présent discours. Ainsi en va-t-il pour le second versant: la "consolation contre la mort" s'oppose à une conception négative de la mort.

La critique s'est déjà attardée à propos du Mespris à observer comment "le locuteur chang[e] la connexion habituelle entre le signifiant 'mort' et le signifié 'mort' afin d'en adoucir la représentation." 16 Néanmoins, s'il est de prime abord frappant que la mort soit, dans ce système renversé, la vraie vie, et la vie sur terre, une agonie éprouvante (le sonnet CLV du Mespris nous dit que la vie de l'homme est "un ouvrage inutile"), il est cependant remarquable que le transfert vie $=$ mort $/$ mort $=$ vie n'est pas l'aspect sémantique le plus particulier du Mespris. En fait, ce qui est connoté négativement, ce n'est pas la vie en soi, qui est dans plusieurs endroits du recueil peinte avec un éclat certain, mais c'est la vie de l'homme:

Les poissons escaillez aiment les moites eaus,

[...]

Les grillons babillars aiment l'email des preaus

[...]

Bref, naturellement chacun aime et desire

Le lieu originel d'où sa naissance il tire [. . . ]

L'homme seul, [... .] 
Prefere à sa patrie [le "ciel spirituel"] un long banissement

(Mespris, CCXL)

et le monde dans lequel il vit; en ce sens, le locuteur emploiera à plusieurs reprises "mondain" (de mondanus) afin de qualifier l'homme: c'est contre celui qui vit attaché au monde, le "sot eshonté qui ne s'estime [mortel]" (Mespris, LXXXVIII), que se dresse ce discours-sermon. Suivant ce raisonnement, non seulement faudrait-il que l'homme vive "comme mortel," mais qu' il vive comme mort, espérant constamment en "la seurté du port" (Mespris, LXVI). Les carrés regroupés ici ${ }^{17}$ illustrent de manière narrative ce cadre axiologique:acabre)]

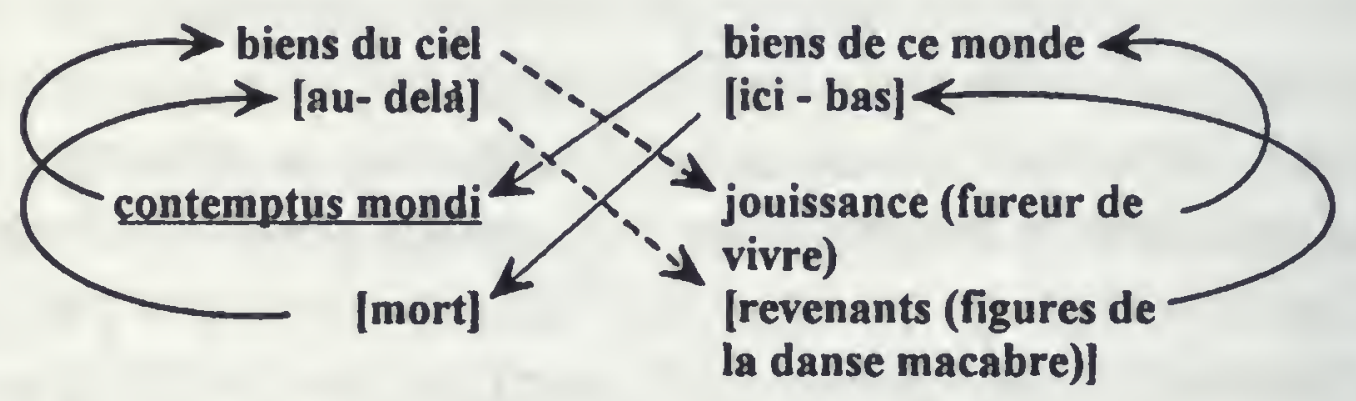

Le parcours à lignes continues (de "biens de ce monde" vers "biens du ciel") indique sur un plan narratif le chemin de l'homme qui souhaite atteindre aux biens du ciel (voir la note 17 au sujet du parcours pointillé). Non seulement une telle doctrine impose-t-elle de désirer la mort, mais elle implique que pour accéder à l'union divine, il est nécessaire de mourir au monde, de cesser d'être classé parmi les vivants:

[...] le monde decevant [...]

[...] chasse loing de soy ceus, mourant en Dieu, Marchent selon l'esprit et vivent en tout lieu

Contemplant nuit et jour l'éternité promise.

(Mespris, CCCXLVIII)

Pourtant, ce discours n'appelle pas le passage par la mort pour atteindre ce but. En effet (et il s'agit d'un héritage patristique ${ }^{18}$ ), le désir de la mort, assimilé au martyre du Christ, n'est plus une envie de mourir exprimant une volonté d'abréger les épreuves terrestres. Et sur ce point, il appartient au domaine de la vérité, dans le Mespris, que l'homme ne peut lui-même se donner la mort afin d'aller rejoindre Dieu. Mourir ne se présente donc pas véritablement comme le /faire/ à accomplir afin d'atteindre aux biens du ciel: c'est en fait une forme de /vouloir mourir/ qui tient la place du /faire/.

Toutefois, sur le plan idéologique, cette "certaine permanence du Moyen Age et du gothique"19 à la fin du seizième siècle se voit confrontée, 
sur le fait que l'homme ne détient pas de pouvoir sur sa vie et n'est en réalité qu'un être en attente d'une mort salutaire, à une autre influence importante qui sillonne partout le recueil de Chassignet: le néo-stoïcisme de la fin du seizième siècle. De là l'impression que le Mespris renferme plusieurs contradictions. En effet, alors que les stoïciens de l'Antiquité "affirment [. . . ] que le sage doit se donner la mort pour sauver sa liberté," 20 les néo-stoïciens, même s'ils valorisent le volontarisme au même titre que leurs maîtres, ${ }^{21}$ n'accordent jamais à l'homme le pouvoir de se donner la mort. Sur le plan du suicide, cette pensée se voit donc tronquée de la modalité de /pouvoir/ qui lui est inhérente:

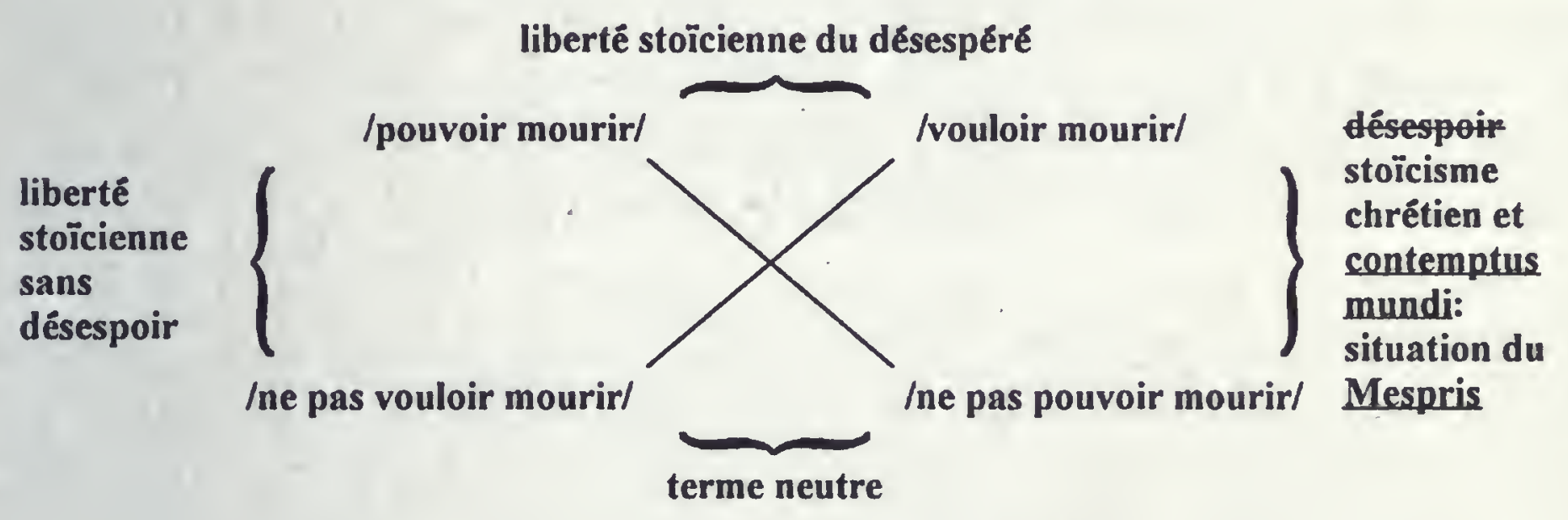

Si j'ai barré le terme désespoir (qui forme la jonction entre /vouloir mourir/ et Ine pas vouloir mourir/), c'est que les stoïciens chrétiens (dont le Montaigne du chapitre "Coustume de l'Isle de Cea" fait partie) considèrent le suicide comme un acte lâche et, bien évidemment, hautement répréhensible. Ainsi que l'écrit Philippe Du Plessis-Mornay dans son Discours de la vie et de la mort:

Bien devons nous tascher de faire mourir nostre chair en nous et en arracher le monde, mais de nous arracher du monde, il ne nous est aucunement permis. Le Chrestien [. . . ] ne doit pas laschement fuir [ceste vie]. ${ }^{22}$

S'il fallait considérer avec modération l'équation un peu simpliste mort = vie, l'équation vie = mort demande la même attention, puisque le locuteur des sonnets à la troisième personne affirme que de "prouffiter à tous, à personne ne nuire, / Parce qu'il n'y a mort qui se passe en martire / Hormis celle où la vie est sans utilité" (Mespris, CCLXXVIII) est la condition d'une mort sereine. Ce discours s'inscrit véritablement contre celui sous-entendu par l'aspect polémique du titre Mespris de la vie. En effet, proposer de mépriser la vie et avancer qu'elle doit être utile, voilà qui peut être considéré comme une position des plus inattendue, voire contradictoire, si l'on omet 
de contextualiser les conditions de production du Mespris. Il arrive donc de rencontrer chez Chassignet, sur le mode de la vérité, un discours visant à valoriser la vie.

\section{La corrélation de subjectivité 23}

C'est d'ailleurs la plupart du temps dans la prétendue authenticité de ce discours que le locuteur ira stratégiquement puiser pour s'allier ses allocutaires, dans les poèmes articulés autour de la fonction conative (où prime l'usage de "tu," "vous" et "nous"). Mais avant de poursuivre, voyons quelle place occupe cet allocutaire en apparence absent dans les pièces qui tiennent lieu d'énoncés de vérité.

La théorie de l'énonciation nous dit qu'il suffit que le sujet énonçant avance des propositions sans marques déictiques "pour que tout allocutaire soit soumis à la même obligation que lui: croire sans réserve." 24 C'est bien ce que l'on peut observer dans l'oeuvre étudiée, où le lecteur, afin de ne pas être identifié ou délocuté, c'est-à-dire celui dont on parle, le "fol" qui "après le jour dernier pense en rien devenir" (Mespris, CLXXXIV) ou le "sot ignorant mourant desesperé" (Mespris, CCXI), qui croient l'un et l'autre que mort = mort, doit savoir que la mort est en fait la vraie vie, et il croira en cela la seule parole du locuteur: "Venez à gorge ouverte en l'eau de mes discours," nous dit ce locuteur conscient du pouvoir de sa parole, "Puiser contre la mort un asseuré secours, / Remettant en DIEU seul vostre unique esperance" (Mespris, I). Le sujet énonçant occupe ici une position forte, puisqu'il se donne la fonction de Destinateur, d'actant "communiqu[ant] au Destinatairesujet [. . . ] l'ensemble des valeurs en jeu," 25 et ce, dans le but manifeste de l'influencer. ${ }^{26}$ En ce sens, il est intéressant de constater de quelle manière sera récupéré, dans les pièces où prédomine la fonction conative, le contenu du discours tenu à la "non-personne": certains cadres axiologiques seront ainsi convoqués tour à tour selon le choix du destinataire, évidemment dans le but d'efficacité qui semble être une caractéristique fondamentale de la littérature baroque: l'adaptation au destinataire. De cette façon, le locuteur opère le transfert de la modalité du /devoir/ qui distingue les pièces rédigées à la troisième personne - où le référent désigné est toujours en dehors de l'acte d'énonciation et désigne les bases d'un /savoir/ - vers le sous-groupe qui nous intéressera ici:

Celuy tousjours à ses jours met la dernière main,

Celuy franc des soucis du tardif lendemain

$\mathrm{N}$ 'a que faire du tems, mais le tems il amende.

(Mespris, XXVI) 
Mais ne presume point d'avancer ta carriere

Ou, comme il te plaira, te tirer en arriere:

La conduite du vent n'est pas en ton pouvoir.

(Mespris, XXVII)

En réitérant le carré ci-haut illustré, c'est évidemment au délocuté du premier tercet que le second est destiné: cette sorte de mise en accusation ou de procès d'intention de l'allocutaire engendre des situations où le locuteur s'adresse d'une position de supériorité à son allocutaire, posant son discours littéralement contre celui de l'autre, dont il suppose qu' il "crain[t]" la mort et que, "mondain," il vit de "voluptez." Son but est bien d'imposer ici l'axiologie du contemptus mundi: la "violence dure" de la mort est en fait une voie pour se libérer du péché, et une joie de vivre trop affichée est le signe d'une trop grande acceptation du monde. Cependant, le locuteur, fin "manipulateur" (son rôle de Destinateur prend ici toute sa signification), exploite le néo-stoïcisme comme philosophie de l'homme dans le monde et investit le champs des valeurs humanistes dans le seul but de s'allier ses allocutaires.

L'usage que fait le locuteur de "tu" ou de "vous," pronoms qui visent la distanciation des actants du discours, est relativement typé. Et il ne suit pas, comme on pourrait le penser d'une oeuvre de cette époque et de cette inspiration, le schéma allocutoire asymétrique non réciproque, mais un schéma symétrique fondé sur la réciprocité, où le tutoiement est synonyme de solidarité, le vouvoiement signifie distance sociale.

Voyons d'abord la relation interpersonnelle "je"/"tu," relation qui vise une certaine particularisation du discours:

Qu'est-ce que vostre vie? une bouteille molle [...]

Qu'est-ce que vostre vie? un mensonge frivole [...]

Qu'est-ce que vostre vie? un tourbillon roüant

De fumiere [...].

(Mespris, XCVIII, je souligne)

Desires tu sçavoir à quoi je paragonne

Le fuseau de tes ans? au saon blanchissant

Soufflé par un tuyau de paille jaunissant

Dont un fol enfançon ses compagnons estonne;

(Mespris, XCIX, je souligne)

Ces extraits sont tirés de deux sonnets qui se suivent dans le recueil (XCVIII et $\mathrm{XCDX}$ ), ce qui rend le contraste encore plus évident: alors que dans le premier (au "vous"), le locuteur répond directement à la question "Qu'est-ce que vostre 
vie?", dans le second (au "tu"), il demande à son allocutaire s'il "desir[e] [. . . ] sçavoir" à quoi il compare ("paragonne") sa vie ("le fuseau de [ses] ans" remarquons l'utilisation d'une métaphore, qui implique une plus grande attention portée à l'allocutaire).

En plusieurs autres endroits du recueil, il est manifeste que cette manière — rapport d'égalité plutôt stratégique - vise à dissocier l'allocutaire de la masse: le locuteur s'adresse à un homme qui lui ressemble, éclairé, qui "tant lis et vois," qui "tant de choses ois" (Mespris, sonnet non numéroté, p. 436). Le lecteur visé y est distingué des mondains, "pervers et malins," et, justement pour ces raisons, se doit d'être sensible à "l'ineffable merveille" de Dieu. Contrairement au mondain ou au pécheur, sa vie n'est pas que mort (quasi absence de contemptus mundi), mais lieu d'accomplissement, car l'homme de génie, même s'il est "hors du monde" - comme nous pouvons le lire dans la "Preface au lecteur"27 — doit acquérir gloire et renommée: "tout ce que ceste vie a de plus convenable, / Embrasse le et le gouste, [. . .]" (Mespris, XXIII). Nous sommes loin du premier schéma que nous avons esquissé plus haut (qui décrit le parcours des biens du monde aux biens du ciel), et plus près de l'esprit d'un texte comme le De tranquillitate animi de Sénèque, où il est entendu que l'homme doit, pour vivre en constance, accepter le monde. Ne nous illusionnons pas cependant; le locuteur, malgré cet apparent accord avec les valeurs qu'il propose à son allocutaire, conserve encore la position de supériorité qui lui est conférée par ce rôle de possesseur du savoir: "Tu verras, dit-il à un certain Casanat, non comme tu le pense (Mespris, LI, je souligne), un savoir qui doit être reçu comme une certitude indéniable. Comme dans les pièces rédigées autour de la troisième personne, l'allocutaire, non plus afin de ne pas se retrouver dans la sphère du délocuté, mais pour éviter d'être visé par le propos tenu, doit reconnaître la validité de ce savoir, comme il est sous-entendu à la fin du Mespris, dans un sonnet s'adressant à un "[m]al-avisé Mondain," où le locuteur-Destinateur sanctionne par avance la transformation opérée par la transmission de l'objet /savoir/:

Estant enfant du monde et suivant le fol monde,

Du monde environné, qu'esperois tu du monde

Que regret, repentir, fascherie, et dedain?

(Mespris, CCCLXVII)

Si l'usage de "tu" vise la particularisation du discours, celui de "vous," pour sa part, a au contraire pour résultat d'augmenter la distance entre le locuteur et l'allocutaire. "Tu ignores ce que je sais" peut signifier qu'un autre 
Isabelle Lachance / A défaut de dire tout: dire partout. Étude des modes énonciatifs / 43

"il" le sait; mais "vous êtes ignorants" reste sans équivoque. . . Ceux que ce pronom désigne ne peuvent jamais fuir la situation d'énonciation dans laquelle ils sont impliqués:

Avortons mal-heureus qui, dez le tendre bers

Tombez dans le tombeau, nourriture des vers,

Comme est ce que l'estat du monde vous contente?

(Mespris, CCCXXXIII)

Car "vous," c'est "tous les autres sauf moi,"28 moi qui sais ce qu'est le monde. Ainsi, lorsqu'il est adressé à des destinataires indifférenciés, l'exercice de ralliement accompli par l'articulation du discours autour de "tu" n'a plus cours. Notons enfin que l'impression d'éloignement fondée sur l'utilisation du pronom "vous" atteint un sommet inégalé dans l'utilisation d'apostrophes aussi sévères qu'offensantes; cette distance avait d'ailleurs été établie dès le "Sonnet premier," destiné à "VOUS quiconque allechez des voluptez charnelles," et atteint son paroxysme à travers cette joliesse baroque:

Hydropiques enflez que le dypsade mord,

Vous plairez-vous ainsi à boire votre mort,

Traittant avec l'enfer une ingratte alliance?

(Mespris, CCCLXXI)

Voyons maintenant quel usage le Mespris de la vie et consolation contre la mort fait du pronom "nous." Puisque ce pronom place le locuteur au centre de son système de valeurs, il s'avère donc possible de poser que les poèmes qui en présentent un usage généralisé impliquent, parce que le locuteur se donne désormais comme faisant partie du même monde que ses allocutaires, "la mise en oeuvre de négociations implicites," 29 comme le porte à croire cet extrait du "Discours a Leonel Fauche," où prime la modération:

Allons par le milieu, fuyant l'extremité,

Alors nous garderons la mediocrité.

Sur tout ne soyons point aus douleurs immobiles,

Ne soyons pas aussi aus douleurs trop faciles,

Mais si nous nous monstrons hommes en soupirant,

Demonstrons nous Chrestiens au moins en moderant

Nos ennuis et regrets, [. . .].

(Mespris, v. 214-219, p. 422)

Il est manifeste ici que le stoïcisme se voit refusé partiellement au profit d'une certaine valorisation de la ferveur religieuse, puisque "contrairement au stoi-cisme, le christianisme n'a jamais fait de la souffrance [.. .] une adiaphora, une chose indifférente." ${ }^{30}$ Ainsi, l'impératif "Demonstrons nous Chrestiens" illustre 
véritablement la position modérée de ce stoïcisme dont les enjeux ont déjà été schématisés. En effet, nous avons vu que le chrétien doit /vouloir mourir/ (au sens de "mourir au monde") afin d'échapper à l'emprise du monde; cependant, puisque dans le Mespris, la doctrine du contemptus mundi se trouve confrontée au renouveau du stoïcisme, qui vise certainement à éloigner l'homme de ses passions (dont fait évidemment partie la passion de l'union divine), le terme se trouvant à la rencontre de /vouloir mourir/ et de /ne pas vouloir mourir/ ne sera pas le désespoir, mais une sorte de flou idéologique dont les principes oscillent entre un contemptus mundi où il est nécessaire de vivre et un stoïcisme qui doit fléchir sa raison aux passions de la foi; il faut vivre dans le monde, comme malgré soi "en moderant / Nos ennuis et regrets."

Toutefois, ce propos nuancé ne perdurera pas jusqu'à la fin du Mespris, et la négociation, véritable marchandage idéologique ici, est bien ce qui caractérise l'utilisation du pronom "nous": le locuteur proposera d'abord un système de valeurs qu'il partage avec son allocutaire, pour ensuite soutenir, sous le couvert de la parole commune, les positions les plus radicales du Mespris telles que nous les avons vues jusqu'ici: valorisation de la mort et dépréciation de la vie.

Je m'attarderai sur des exemples qui concernent l'oeuvre de l'homme, son savoir et la gloire ou la renommée qu'il peut en tirer:

Par le labeur d'autruy nos languissans espris

Sont guidez et conduis à chose de grand pris

Qui nous donne par tout une libre avenue

Et, si nous surmontons nostre imbecillité,

Nous avons assez tems pour rendre plus connus

De nos espris divins la magnanimité.

(Mespris, LXXVII)

La culture humaniste, qui prévalait encore au temps de Chassignet (cet extrait en témoigne), soutenait fermement que l'acquisition de connaissances élevait l'âme et qu' au contraire, l'ignorance constituait presque un vice, ne serait-ce que parce que ces connaissances portent sur "l'ineffable merveille" de Dieu. La valeur du savoir de l'homme sur le monde n'était l'objet d'aucun doute et, stratégiquement, notre locuteur emprunte d'abord plus volontiers la position d'un Louis le Roy qui, dans De la vicissitude ou varieté des choses en l'univers, dit qu'“à l'exemple [des Anciens et des hommes qui nous précèdent] devons [. . . ] travailler avec esperance de nous rendre meilleurs qu'eux: aspirans tousjours à perfection," "31 que celle de Cornelius Agrippa dans De la vanité de tout savoir et 
de l'excellence de la parole de Dieu (qui aurait pourtant mieux concordé avec les "vérités" soutenues dans Le mespris).

Cependant, une fois pénétrée la sphère éthique de ses allocutaires et le "dialogue" établi, il s'acharnera à détruire ces certitudes en remettant en cause les principes mêmes dont il a exposé la grandeur, notamment en y appliquant le postulat de l'Ecclésiaste, dont l'art baroque a fait un leitmotiv:

Las! de quoy nous sert il que nos rares espris

Comprenant des haus cieux la plaisante harmonie

Et les cercles mouvans de leur voute infinie?

(Mespris, “Oraison à Dieu," pp. 521-522)

Un tel discours révèle certainement un parti pris contre 1' "orgueil" des humanistes, suivant directement la tendance des autorités religieuses catholiques pour qui "la foi en l'homme est sacrilège." 32 Dans un tel retournement des valeurs (par rapport à l'humanisme chrétien), l'homme ne peut aspirer qu'à une seule chose: s'être suffisamment préparé à la mort, avoir su l'attendre et considérer le reste comme accessoire. C'est dire qu'une mort ainsi attendue, espérée, devra se voir débarrassée de toute connotation négative.

Toujours en structurant son discours autour de cette première personne du pluriel unificatrice, le locuteur réussira à "trafiquer" son dire, et si les lecteurs ont pu croire qu'en s'intégrant dans les objets de son discours, il l'adoucirait au profit d'une certaine acceptation du monde, ils ont tôt fait de s'apercevoir qu'en fait le "nous" en vient à servir de médiateur pour prononcer le même enseignement que partout ailleurs. L'allocutaire doit penser (et dire lui-même) ces vérités: "nous" est devenu un "je" décuplé à l'infini:

Chantons comme l'oyseau du Meandre agité,

Voyant venir le jour du trespas souhaitté,

Disant avec David: - ò mon Dieu, mon attente.

(Mespris, "A Antoine Huet, principal du college de Besançon," p. 466).

\section{L'usage exemplaire ${ }^{33}$ de la subjectivité dans le Mespris}

Mais qui est ce "je" qui finit toujours par promouvoir les valeurs du contemptus mundi, malgré toutes les finesses de son argumentation? Il est loin d'être, comme le "je" chez Montaigne (pourtant une source constante pour Chassignet), une "médiation pour connaître le monde." 34 Il est en fait une subjectivité montrée au service de l'illustration (suivant ainsi la volonté exprimée dans la "Preface au lecteur") et le véhicule d'un discours exemplaire (la modalité du /devoir/ surplombe également cet aspect du Mespris). 
A ce jour, on a considéré ce "je" comme référant directement à la personne de Jean-Baptiste Chassignet. Dans sa thèse récente sur Chassignet, Roland Guillot soutient que lorsqu'il est plus enclin à centrer son discours autour d'une subjectivité forte, à parler de lui-même, à faire état des fluctuations de son âme, de sa foi, ce "je" forme le "double inversé" d'un autre, "idéal"35 qui a pour rôle de dispenser un enseignement en forme de sermon. Il me paraît sinon douteux, du moins discutable que l'auteur de cette thèse, qui accorde par ailleurs autant de crédit au génie créateur (il nous dit que le poète, "créateur de métaphores" est "vraiment démiurge" 36 ), s'acharne à débusquer les mécanismes inconscients qui auraient fait surgir ces deux modalités d'un même sujet de l'énonciation (dont le "moi," évidemment, serait "clivé" 37 ), au lieu de relier cette exposition d'une subjectivité à la volonté de Chassignet d'illustrer son propos. Pourtant, il est clair que les nombreux modèles mis à profit par cet auteur, religieux ou autres, simples influences ou parcours nettement calqués, contribuent à la construction d'une persona typiquement littéraire et non à l'expression d'un quelconque moi original ou artiste: on semble avoir oublié qu'à la Renaissance la singularité du poète tire son origine d'un "moi construit," forme d" "exhibition rhétorique de soi."38

Cette "exhibition" est à rattacher spécifiquement, dans le Mespris de la vie et consolation contre la mort, à l'usage de l'exemplum dans le sermon religieux: en effet, à la fin du seizième siècle, sous l'influence du prêche réformé, cette pratique a évolué vers l'emploi de "l'histoire individuelle" comme référence à "l'enseignement spirituel"39: "le récit de l'expérience individuelle vise ainsi à créer les conditions d'une expérience analogue pour autrui." 40 De plus, il est remarquable que cette stratégie ne soit pas unique à Chassignet: Ignace de Loyola avait déjà rédigé certaines parties de ses Exercices spirituels à la première personne. Ainsi, l'auteur des Exercices ne tient pas compte de l'identité réelle de son lecteur: il exige qu'il reconnaisse avoir commis de "grands" et "nombreux" péchés:

Lorsque je me réveillerai, j'éloignerai de mon esprit toute autre pensée, pour m'occuper de suite du sujet que je dois méditer dans le premier Exercice qui se fait au milieu de la nuit, $m$ 'excitant à la confession de mes péchés, si grands et si nombreux, ${ }^{41}$

comme le locuteur du Mespris le fera à son tour dans l'introduction d'un poème rédigé à la première personne, la "Synderese a Dieu tout-puissant" (Mespris, p. 117), "propre à tous ceus qui, mordus en leur conscience et touchez du repenti de leur fautes, se retournent à la misericorde de Dieu": 
Depuis le gouffre obscur

De mes ennuis, au fort de tant d'alarmes, Je veux enfler de sanglos et de larmes

Et mes yeus et mon cueur;

(Mespris, "Synderese a Dieu tout-puissant," p. 117).

Le lecteur du Mespris est donc placé devant une oeuvre qui n'hésite pas à doubler le point de vue, afin de rendre compte de la multiplicité du monde et de ses mouvements divers, selon l'un des fondements du baroque: cette composition répond ainsi directement aux principes de variété, d'ornement et de recherche artistique capables d'exciter la foi et la passion. Un court aperçu d'une autre "Synderese" (Mespris, p. 192) nous aidera à saisir cette sorte de glissement énonciatif:

Note introductive

CESTE SYNDERESE fait voir à l'oeil en quelle confusion languit le pecheur qui, se deffiant de la misericorde du pere celeste adresse sa plainte aus hommes, ausquels il n'y a ny constance ny salut.

1. N'est-il pas malheureux

Qui tourmenté d'une forte detresse,

N'espère rien au fort de la tristesse

Qu'un trespas rigoureus (v. 33-36)?

2. Ainsi de sa fureur

Prend son repos le troublé frenetique, Ainsi se plaît le cerveau fantastique

De nourrir son erreur (v. 53-56).

Les extraits 1 et 2 forment en fait des interventions du locuteur tel que nous l'avons vu jusqu'ici dans le corps même du discours de celui dont il parle, le "pecheur," et réitèrent la distance établie dès la note introductive. De la même manière, si l'homme doit /vouloir mourir/, il ne doit pas inévitablement désirer la mort pour atteindre à l'union divine, suivant l'application la plus stricte au christianisme de la théorie platonicienne. C'est pourtant ce que fait le locuteur de cette "Synderese" (le sujet "exemplaire," montré) en interpellant la "Parque" ou la "Mort" à son secours. Le sens de "malheureux," que l'on doit prendre ici dans son acception presque esthétique de "médiocre," est également à rapprocher du "fol" ou "sot ignorant" que nous avons rencontré plus tôt. Ces qualificatifs s'appliquaient bien sûr à l'homme qui ne croit pas ou qui ne "sait" pas les "vérités" énoncées à plusieurs reprises par le locuteur principal, signifiant qu'une forme de/vouloir mourir/ (dans un contemptus mundi que le substrat néo-stoïcien 
dégage de l'obligation de mourir, même seulement de "mourir au monde") se substitue au /faire/ ("mourir"), puisque Dieu seul a le/pouvoir/ de donner la mort. Dans l'extrait 2, un vocabulaire pseudo-médical - "fureur," "frenetique," "cerveau fantastique" - se voit affublé d'une connotation morale ("nourrir son erreur"): n'oublions pas que selon le système développé dans les poèmes à la "non-personne," le /savoir/ s'articule autour de la modalité du /devoir/.

Et en quoi ce second sujet de l'énonciation "erre"-t-il? Sa prière s'adresse non pas à Dieu, mais à des hommes - ses "chers amis" - il ne demande pas le salut de Dieu, mais celui d'une mort dénuée de tout sens chrétien (la "Parque"). Or il est frappant, à la lecture d'une recommandation que le locuteur principal adresse à un "MORTEL" au sonnet CXXXV, qu'il dirige son discours spécifiquement vers cet autre sujet de l'énonciation du Mespris:

Fonde en DIEU seulement, estimant vanité

Tout ce qui ne te rend plus sçavant et plus sage.

Comme nous l'avons vu plus tôt, le locuteur de la "Synderese," le "pecheur," place son attente dans un sujet opérateur (ou actant chargé de le conjoindre à l'objet-valeur "mort") autre que Dieu: il ne "fonde" pas "en Dieu seulement": c'est donc à lui que s'adressent les deux vers cités plus haut.

A la manière d'un récit, et après de multiples oscillations et variations (concentrées, comme l'exemplum dans le sermon, à la fin du recueil), le "je" pécheur finira par se convertir aux "vérités" et impératifs du locuteur principal (ce "je" incarne en fait le "tu" de plusieurs sonnets) et mettra son attente en Dieu; ainsi son discours sera-t-il formulé sous forme de prière: plusieurs sonnets posent un sujet d'état en conjonction avec le mal, implorant Dieu de l'en défaire. A la fin de cette "histoire," l' "Oroison a dieu tout-puissant," dernier sonnet du Mespris, résoud le parcours tracé plus haut (des "bien de ce monde" [ici-bas] aux "biens du ciel" [au-delà], en passant par le rejet du monde ou contemptus mundi [mort]). Mais l'on connaissait dès le début du recueil, comme une thèse de départ, ce dénouement en forme de conversion. En effet, on remarquera, et j'achèverai cette étude sur ce commentaire un peu opportuniste, que Chassignet réussit à conjoindre un modèle religieux à un modèle de persona poétique, justifiant ainsi le caractère sombre du poète mélancolique et solitaire (suivant le modèle pétrarquiste) par la nécessité de rester hors du monde:

Oui, C'est Chassignet tant amoureus jadis, Jadis si pres du monde et loing de Paradis,

Qui vit encore au monde et du monde se fache. 
Il n'est pas deffendu au pecheur se sentir

Sous un visage gay un juste repentir.

Le vray remord du cueur au cueur mesme se cache.

(Mespris, "Oroison a dieu tout-puissant,")

Le fait est que la doxa littéraire, par sa valorisation du tempérament mélancolique, "allait directement à l'encontre de l'enseignement traditionnel de l'Église romaine représentant l'acedia ${ }^{42}$ comme un péché capital," 43 le retrait du monde et l'abandon de l'être à la passion comme autant de maux à combattre pour une Église qui entretenait des liens politiques fermes avec le pouvoir. ${ }^{44} C^{\prime}$ 'est entre autres raisons pour affirmer cette position que le christianisme de la fin du seizième siècle a pu prendre des allures stoïciennes.

Chassignet, dans sa "Preface," nous dit de son oeuvre que ses "tissures" sont "si mal jointes et unies que les liaisons et coustures y parroissent comme les veines, les nerfs, et les os dans un cors maigre, have et deffait" (Mespris, p. 17). A cette piètre (et presque triviale) opinion qu'il a de son travail, nous pourrions aisément rétorquer que par les diverses stratégies discursives qu'il a su mettre en place en s'assurant que toutes les positions énonciatives y soient occupées et en doublant le point de vue afin de répondre aux principes baroques de variété, d'ornement et d'appel à la passion, son Mespris jouit d'une valeur de persuasion presque à toute épreuve. Il le fallait bien, puisqu'il se trouverait sûrement devant son livre quelque esprit plus libre pour lui remettre avec Montaigne que "toutes les opinions du monde en sont là, que le plaisir est nostre but [.. . ]; autrement, on les chasseroit d'arrivée, car qui escouteroit celuy qui pour sa fin establiroit notre peine et mesaise?"45

\section{Université Laval}

\section{Notes}

* Ce texte a d'abord été présenté dans le cadre du congrès annuel de la Société Canadienne d'Études de la Renaissance (Memorial University of Newfoundland, juin 1997).

1. Ilana Zinguer, "Auto-constitution: aspects de la subjectivité chez Montaigne," in La problématique du sujet chez Montaigne, sous la direction de Eva Kushner (Paris, Champion, 1995), p. 59.

2. Tel est le titre du chapitre que consacre André Gabriel au Mespris de la vie dans sa thèse "L'inconscient dans la poésie baroque," University of California, Berkeley, 1976.

3. Roland Guillot, "Le Mespris de la vie et consolation contre la mort de Jean-Baptiste Chassignet: une parole divisée," thèse de doctorat nouveau régime, Université de Paris VIII, 1990, pp. 226 et 273. 
4. François Ruchon, "Jean-Baptiste Chassignet: Le Mespris de la vie et consolation contre la mort," Bibliothèque d'Humanisme et Renaissance, XV (1953), p. 58.

5. Tibor Klaniczay, "La théorie esthétique du maniérisme," in La littérature de la Renaissance à la lumière des recherches soviétiques et hongroises (Budapest, Akadémiai Kiadó, 1978), p. 328. Nous ne contredisons pas les avancées de cet auteur au sujet du maniérisme, puisqu'il ne fait pas référence à l'oeuvre étudiée ici.

6. Jean-Baptiste Chassignet, Mespris de la vie et consolation contre la mort, édition établie et préfacée par H.-J. Lope (Genève, Droz, 1967). Référence sera faite dans le texte à cette édition, suivie de la page.

7. R. Guillot, p. 18.

8. Tibor Klaniczay, p. 371.

9. Thomas Sébillet, Art poétique françois, édition établie par F. Goyet (Paris, Le Livre de Poche, 1990), p. 57.

10. François Cornilliat, "Or ne mens." Couleurs de l'éloge et du blâme chez les grands rhétoriqueurs (Paris, Champion, 1994), p. 12.

11. Claude Calame, "Le sujet de l'énonciation: quelques réflexions liminaires," Cahiers du département des langues et sciences du langage, 4 (1987), p. 8.

12. Catherine Kerbrat-Orecchioni, Les interactions verbales, t. 1 (Paris, Armand Colin, 1990), pp. 9-10.

13. Terence C. Cave, Devotional Poetry in France (1570-1613) (Cambridge, Cambridge University Press, 1969), p. 152.

14. A ce propos, voir Juste lipse, De la constance, traduction et introduction de Lucien Du Bois (Bruxelles/Leipzig, Henry Merzbach Éditeur, 1873), p. 117: "J'ai cherché des consolations contre les maux publics [. . . ]. Je suis de ceux qui ont la piété dans le coeur plus que sur les lèvres; $\mathrm{j}$ 'aime mieux la pratiquer dans mes actes, que d'en faire montre dans des paroles. [. . . ] Et, au bout du compte, quand tous les disputeurs ont ainsi parcouru le ciel et la terre sur l'aile d'un génie subtil, qu'ont-ils fait de plus que de marcher dans les nuées [...]"'(Ibid., p. 127)? Voir également Philippe Du Plessis-Mornay, Discours de la vie et de la mort, édition et préface par Mario Richter (Milan, Editrice Vita e Pensiero, 1964), p. 60: "[. . .] que tel fait profession de fuir le monde qui cherche par là la louange [. . . ]: tel fait semblant de fuir." C'est le Moyen Age qui alliera plus étroitement l'ascèse à la sagesse stoïcienne. L'usage que l'on faisait alors des préceptes de Sénèque et de ses disciples s'avérait tout à fait différent de celui qu'en feront les néo-stoïciens, dont la morale est davantage sociale ou politique. Cf. Léontine Zanta, La renaissance du stoïcisme au XVI siècle (Paris, Champion, 1914), pp. 98 et ssq.

15. Jean Cervoni, L'énonciation (Paris, Presses Universitaires de France, 1987), notamment pp. 33 et ssq., a bien su éclairer cette problématique, et j'adhère volontiers à sa position modérée concernant la présence de la subjectivité dans le langage: en effet, cet auteur, prenant ses distances par rapport à un certain courant voulant que tout discours soit subjectif du fait qu'il soit articulé par une personne, exclut le discours à la non-personne de la deixis.

16. Roland Guillot, p. 69.

17. Ils sont tous deux tirés de Joseph Courtés, "Sémiotique et théologie du péché," in Herman Parret et Hans-George Ruprecht, Exigences et perspectives de la sémiotique: recueil 
Isabelle Lachance / A défaut de dire tout: dire partout. Étude des modes énonciatifs / 51

d'hommages pour Algirdas Julien Greimas, t. 2 (Philadelphie, John Benjamins, 1985), 863-903. Nous ne nous attarderons pas aux parcours pointillés, qui concernent plus spécifiquement le motif des "danses macabres." En effet, comme le montre Courtés, il y a un parallèle flagrant entre un parcours qui mènerait du rejet des biens du ciel à l'atteinte des biens du monde en passant par la jouissance et la figure du revenant. C'est pour tenter de contrer cette utilisation plutôt épicurienne de la danse macabre que l'on a utilisé, au Moyen Age et encore au seizième siècle, ce motif dans les sermons religieux. Nous ne pouvons affirmer que le Mespris témoigne de cette pratique.

18. Voir entre autres Augustin, La Cité de Dieu, livre XIII ("Chute et destinée de l'homme").

19. André Chastel, "Le baroque et la mort," in Fables, formes, figures, t. I (Paris, Flammarion, 1978), p. 210.

20. Jacqueline Lagrée, Juste Lipse: la restauration du stoïcisme (Paris, Vrin, 1994), p. 110.

21. A ce sujet, voir J. Lagrée, pp. 78 et ssq. Également, Montaigne, Essais, livre II, chap. III ("Coustume de l'Isle de Cea"), édition établie par Albert Thibaudet et Maurice Rat (Paris, Gallimard, 1962), pp. 332-333: "[. . . ] car plusieurs tiennent que nous ne pouvons abandonner cette garnison du monde sans le commandement exprès de celuy qui nous y a mis; et que c'est à Dieu, [. . . ] de nous donner congé quand il lui plaira [. . . ]. Il y a bien plus de constance à user la chaine qui nous tient qu'à la rompre."

22. P. Du Plessis-Mornay, p. 77, je souligne.

23. L'expression est tirée de Émile Benveniste, Problèmes de linguistique générale, t. I (Paris, Gallimard, 1966), p. 232. Elle désigne toute situation d'énonciation où interviennent "je" et "tu."

24. Jean-Claude Coquet, Le discours et son sujet 1: Essai de grammaire modale (Paris, Klincksieck, 1984), pp. 169-170.

25. Algirdas Julien Greimas et Joseph Courtés, Sémiotique. Dictionnaire raisonné de la théorie du langage, t. I (Paris, Hachette, 1979), p. 95. La majuscule de "Destinateur" est une convention de la sémiotique greimassienne (qui veut ainsi distinguer ce Destinateur du destinateur jakobsonnien (cf. le schéma de la communication) à laquelle Jean-Claude Coquet ne s'est pas conformé: il n'y a donc ici aucune différence de sens entre destinateur et Destinateur.

26. Il me sera donné de revenir longuement sur cet aspect de transmission plus loin, alors que nous verrons que le parcours emprunté par un certain "je" du Mespris de la vie et consolation contre la mort est le résultat direct de cette manipulation (à la fois au sens que confère la sémiotique narrative à ce mot et à celui de "manoeuvre"). Roland Guillot, p. 394, remarque à ce sujet que dans le Mespris, "mis en situation d'élève, traumatisé dans son moi [sic], le destinataire n'a plus qu'à se soumettre au dire magistral dont l'intention première est évidemment didactique."

27. "A ceus qui voudront affirmer que la memoire du trespas peut rarement tomber au sens d'un jeune homme, je maintien qu'il n'y a rien dequoy je me sois tousjours plus entretenu que des imaginations de la mort [. . .]" (Mespris, "Preface au lecteur," p. 15). Voilà comment se décrit le "poète" Chassignet: différent des jeunes gens de son temps et hors du monde. Il est cependant remarquable que l'auteur copie ici intégralement Montaigne, Livre I, ch. XX ("Que philosopher c'est apprendre à mourir"), p. 85: "Il n'est rien dequoy je me soye dès toujours plus entretenu que des imaginations de la mort [. . .]." Mais l'omission que 
Chassignet fait de la phrase qui vient juste avant cette dernière est certainement l'indice qui prouve qu'il a choisi de s'inscrire dans la tradition poétique de son temps, suivant le paradigme de la mélancolie. Montaigne spécifie: "Je suis de moy-mesme non melancholique, mais songecreux."

28. Françoise Madray-Lesigne, "L'ici et l'ailleurs de la personne en discours. Quelques affinités entre personnes, temps et modes en français," in La deixis, sous la direction de Mary-Annick Morel et Laurent Danon-Boileau (Paris, Presses Universitaires de France, 1992), p. 403.

29. C. Kerbrat-Orecchioni, pp. 28-29.

30. Jean-Louis Chrétien, "Pouvoir mourir et devoir mourir selon la théologie chrétienne," Le Temps de la Réflexion, no 3 (1982), p. 85.

31. Louis Le Roy, De la vicissitude ou varieté des choses en l'univers (Paris, Fayard, 1988 [1575]), p. 430.

32. Didier Souiller, "Le paradoxe de la fin du XVI" siècle: décadence et/ou affirmation du baroque," in Fins de siècles: terme - évolution - révolution?, sous la direction de Gwenhaël Ponnau (Toulouse, Presses Universitaires du Mirail, 1989), p. 79.

33. Au sujet de la notion d'exemple (exemplum) appliquée à la poésie de la Renaissance, on référera notamment à Jean-Claude Moisan, "La codification de la passion: Louise Labé (Sémiramis) - Ronsard (Hercule)," in La peinture des passions de la Renaissance à l'âge classique, sous la direction de Bernard Yon (Saint-Étienne, Publications de l'Université de Saint-Étienne, 1995), 111-126.

34. Voir la note 1.

35. Roland Guillot, p. 20.

36. Ibid., p. 206.

37. Voir la note 3.

38. Jean Lecointe, L'idéal et la différence: la perception de la personnalité littéraire à la Renaissance (Genève, Droz, 1993), p. 263.

39. Claude Blum, La représentation de la mort dans la littérature française de la Renaissance, t. II (Paris, Champion, 1989), p. 530.

40. Didier Souiller, p. 84.

41. Ignace de Loyola, Exercices spirituels, traduit de l'espagnol par Pierre Jennesseaux (Paris, Arléa, 1991), p. 112, je souligne.

42. Pour les rapprochements à faire entre mélancolie et acedia, voir Marie-Claude Lambotte, Esthétique de la mélancolie (Paris, Aubier, 1984), pp. 36 et ssq., ainsi que Jean Delumeau, Le péché et la peur: la culpabilisation en Occident (XIII -XVIII siècles) (Paris, Fayard, 1983), pp. 200 et ssq.

43. Marc Fumaroli, "Nous serons guéris si nous le voulons. Classicisme français et maladie de l'âme," Débat, 29 (1984), p. 93.

44. Voir notamment Léontine Zanta, pp. 22 et ssq.

45. Montaigne, ch. XX (“Que philosopher c'est apprendre à mourir"), p. 80. 